\title{
Correction to: Compression of a mixed antiproton and electron non-neutral plasma to high densities ${ }^{\star}$
}

\section{Eur. Phys. J. D 72: 76 (2018), DOI: 10.1140/epjd/e2018-80617-x}

Stefano Aghion ${ }^{1,2}$, Claude Amsler ${ }^{3}$, Germano Bonomi ${ }^{4,5}$, Roberto S. Brusa ${ }^{6,7}$, Massimo Caccia ${ }^{2,8}$, Ruggero Caravita $^{9,10}$, Fabrizio Castelli ${ }^{2}{ }^{11}$, Giovanni Cerchiari ${ }^{12}$, Daniel Comparat ${ }^{13}$, Giovanni Consolati ${ }^{1,2}$, Andrea Demetrio ${ }^{14}$, Lea Di Noto ${ }^{9,10}$, Michael Doser ${ }^{15}$, Craig Evans ${ }^{1,2}$, Mattia Faní ${ }^{9,10,15}{ }^{\text {, Rafael Ferragut }}{ }^{1,2}$, Julian Fesel ${ }^{15}$, Andrea Fontana ${ }^{5}$, Sebastian Gerber ${ }^{15}$, Marco Giammarchi ${ }^{2}$, Angela Gligorova ${ }^{3}$, Francesco Guatieri ${ }^{6,7}$, Stefan Haider ${ }^{15}$, Alexander Hinterberger ${ }^{15}$, Helga Holmestad ${ }^{16}$, Alban Kellerbauer ${ }^{12}$, Olga Khalidova ${ }^{15}$, Daniel Krasnický10,a, Vittorio Lagomarsino ${ }^{9,10}$, Pierre Lansonneur ${ }^{17}$, Patrice Lebrun ${ }^{17}$, Chloé Malbrunot ${ }^{3,15}$, Sebastiano Mariazzi ${ }^{6,7}$, Johann Marton ${ }^{3}$, Victor Matveev ${ }^{18,19}$, Zeudi Mazzotta ${ }^{2,11}$, Simon R. Müller ${ }^{14}$, Giancarlo Nebbia ${ }^{20}$, Patrick Nedelec ${ }^{17}$, Markus Oberthaler ${ }^{14}$, Nicola Pacifico ${ }^{15}$, Davide Pagano ${ }^{4,5}$, Luca Penasa ${ }^{6,7}$, Vojtech Petracek ${ }^{21}$, Francesco Prelz ${ }^{2}$, Marco Prevedelli ${ }^{22}$, Benjamin Rienaecker ${ }^{15}$, Jacques Robert ${ }^{13}$, Ole M. Røhne ${ }^{16}$, Alberto Rotondi ${ }^{5,23}$, Heidi Sandaker ${ }^{16}$, Romualdo Santoro ${ }^{2,8}$, Lillian Smestad ${ }^{15,24}$, Fiodor Sorrentino ${ }^{10}$, Gemma Testera ${ }^{10}$, Ingmari C. Tietje ${ }^{15}$, Eberhard Widmann ${ }^{3}$, Pauline Yzombard ${ }^{12}$, Christian Zimmer ${ }^{12,15,25}$, Johann Zmeskal ${ }^{3}$, Nicola Zurlo ${ }^{5,26}$, and Massimiliano Antonello ${ }^{2,8}$

1 Politecnico of Milano, Piazza Leonardo da Vinci 32, 20133 Milano, Italy,

2 INFN Milano, via Celoria 16, 20133 Milano, Italy

3 Stefan Meyer Institute for Subatomic Physics, Austrian Academy of Sciences, Boltzmanngasse 3, 1090 Vienna, Austria

4 Department of Mechanical and Industrial Engineering, University of Brescia, via Branze 38, 25123 Brescia, Italy

5 INFN Pavia, via Bassi 6, 27100 Pavia, Italy

6 Department of Physics, University of Trento, via Sommarive 14, 38123 Povo, Trento, Italy

7 TIFPA/INFN Trento, via Sommarive 14, 38123 Povo, Trento, Italy

8 Department of Science, University of Insubria, Via Valleggio 11, 22100 Como, Italy

9 Department of Physics, University of Genova, via Dodecaneso 33, 16146 Genova, Italy

10 INFN Genova, via Dodecaneso 33, 16146 Genova, Italy

11 Department of Physics, University of Milano, via Celoria 16, 20133 Milano, Italy

12 Max Planck Institute for Nuclear Physics, Saupfercheckweg 1, 69117 Heidelberg, Germany

13 Laboratoire Aimé Cotton, Université Paris-Sud, ENS Cachan, CNRS, Université Paris-Saclay, 91405 Orsay Cedex, France

14 Kirchhoff-Institute for Physics, Heidelberg University, Im Neuenheimer Feld 227, 69120 Heidelberg, Germany

15 Physics Department, CERN, 1211 Geneva 23, Switzerland

16 Department of Physics, University of Oslo, Sem Sælandsvei 24, 0371 Oslo, Norway

17 Institute of Nuclear Physics, CNRS/IN2p3, University of Lyon 1, 69622 Villeurbanne, France

18 Institute for Nuclear Research of the Russian Academy of Science, Moscow 117312, Russia

19 Joint Institute for Nuclear Research, 141980 Dubna, Russia

20 INFN Padova, via Marzolo 8, 35131 Padova, Italy

21 Czech Technical University in Prague, Břehová 7, 11519 Prague 1, Czech Republic

22 University of Bologna, Viale Berti Pichat 6/2, 40126 Bologna, Italy

23 Department of Physics, University of Pavia, via Bassi 6, 27100 Pavia, Italy

24 The Research Council of Norway, P.O. Box 564, 1327 Lysaker, Norway

25 Department of Physics, Heidelberg University, Im Neuenheimer Feld 226, 69120 Heidelberg, Germany

26 Department of Civil Engineering, University of Brescia, via Branze 43, 25123 Brescia, Italy

Received 9 September 2019

Published online 15 November 2019 - (C) EDP Sciences / Società Italiana di Fisica / Springer-Verlag GmbH

Germany, part of Springer Nature, 2019

\footnotetext{
* The online version of the original article can be found at https://doi.org/10.1140/epjd/e2018-80617-x
}

a e-mail: daniel.krasnicky@ge.infn.it 
This correction provides updated acknowledgements:

This work was supported by Istituto Nazionale di Fisica Nucleare; the Swiss National Science Foundation Ambizione Grant (No. 154833); a Deutsche Forschungsgemeinschaft research grant; an excellence initiative of Heidelberg University; Marie Sklodowska-Curie Innovative Training Network Fellowship of the European Commission's Horizon 2020 Programme (No. 721559 AVA); European Union's Horizon 2020 research and innovation programme under the Marie Sklodowska-Curie grant agreement ANGRAM No 748826; European Research Council under the European Union's Seventh Framework Program FP7/2007-2013 (Grants Nos. 291242 and 277762); Austrian Ministry for Science, Research, and Economy; Research Council of Norway; Bergen Research Foundation; John Templeton Foundation; Ministry of Education and Science of the Russian Federation and Russian Academy of Sciences; and the European Social Fund within the framework of realizing the project, in support of intersectoral mobility and quality enhancement of research teams at Czech Technical University in Prague (Grant No. CZ.1.07/2.3.00/30.0034). 